\title{
Multicentric Plasma-Cell Type Castleman Disease Masquerading As Hodgkin Lymphoma: A Case Report
}

\author{
Timothy Amos Ekwere ${ }^{1} \quad$ Uchechukwu Brian Eziagu²,
}

Address for correspondence Timothy A. Ekwere, MBBCh, MSc, FMCPath, Department of Haematology, University of Uyo/University of Uyo Teaching Hospital, Uyo, Akwa Ibom, Uyo 520001, Nigeria (e-mail: timothyekwere@yahoo.com).

\begin{abstract}
Castleman disease (CD), or angiofollicular hyperplasia, or giant lymph node hyperplasia, is a heterogeneous benign lymphoproliferative disorder of unknown etiology. It has three distinct histologic subtypes (hyaline vascular, plasma cell, and mixed hyaline vascular plasma cell types) as well as unicentric Castleman disease (UCD) and multicentric Castleman disease (MCD) variants. In the unicentric form, the disease is confined to one anatomical lymph node and usually with no systemic symptoms. However, in the multicentric form (further subdivided into idiopathic MCD, human

Keywords

- Castleman disease

- immunohistochemistry

- lymphoproliferative disorders herpes virus-8-associated MCD, and POEMS-associated MCD), lymphadenopathy is more generalized with more aggressive systemic symptoms mimicking a malignant lymphoma. Therefore, this case report aims to underscore the importance of immunohistochemical evaluation as an indispensable ancillary technique to routine histopathological examination of a lymph node biopsy specimen, as a gold standard for definitive diagnosis of proliferative lymph node lesions.
\end{abstract}

\section{Introduction}

Castleman disease (CD), or angiofollicular hyperplasia or giant lymph node hyperplasia, is a rare non-neoplastic lymphoproliferative disorder with variable clinicopathologic subtypes. ${ }^{1,2}$ It was first described by Castleman et al in 1954 in a group of patients with localized lymph node hyperplasia., ${ }^{2,3}$ The etiology of $C D$ remains largely unknown; the pathogenesis show inflammatory response to a mysterious antigenic stimulus with chemical mediators (cytokines) of inflammation particularly interleukin-6 (IL6) playing a prominent role. ${ }^{1,2} \mathrm{CD}$ can be categorized histomorphologically into three distinct subtypes, namely: hyaline vascular, plasma cell, and mixed hyaline vascular plasma cell types. ${ }^{1,2,48} \mathrm{CD}$ also has two clinicopathological presentations, namely: unicentric Castleman disease (UCD) and multicentric Castleman disease (MCD) forms, wherein the unicentric forms are more common. ${ }^{1,25,6,8}$ The UCD form presents

DOI https://doi.org/

10.1055/s-0040-1721158 ISSN 0974-2727. as a solitary (unifocal) lymph node lesion especially in the mediastinum and less commonly in extrathoracic sites and may not be accompanied by systemic symptoms such as fever, night sweats, fatigue, weight loss, splenomegaly, anemia, and hypergammaglobulinaemia. $1,2,5,6,8$ The MCD form, on the other hand, presents as a multifocal lymph node lesion with generalized lymphadenopathy, especially in the neck region, associated with more aggressive systemic symptoms such as fever, night sweats, fatigue, cachexia, splenomegaly, cytopenia, and hypoglobulinemia/hyperglobulinemia, hence resembling a malignant (Hodgkin's) lymphoma. .,25,6,8 $^{1}$ Furthermore, this MCD form can be subcategorized into three subtypes, namely: human herpes virus-8 associated MCD (HHV8-MCD), polyneuropathy, organomegaly, endocrinopathy, monoclonal plasma cell disorder, skin changes-associated MCD (POEMSMCD), and idiopathic MCD (iMCD), 2,6,8,9 Additionally, iMCD is further subdivided into two types, namely: iMCD associated with thrombocytopenia, anasarca, fever, reticulin

(c) 2020. The Indian Association of Laboratory Physicians.

This is an open access article published by Thieme under the terms of the Creative Commons Attribution-NonDerivative-NonCommercial-License, permitting copying and reproduction so long as the original work is given appropriate credit. Contents may not be used for commercial purposes, or adapted, remixed, transformed or built upon. (https://creativecommons.org/licenses/by-nc-nd/4.0/)

Thieme Medical and Scientific Publishers Pvt. Ltd., A-12, 2nd Floor, Sector 2, Noida-201301 UP, India 
myelofibrosis and renal dysfunction and organomegaly (iMCD-TAFRO) and IMCD not associated with TAFRO hence not otherwise specified (iMCD-NOS). ${ }^{2,8}$ It is also noted that nonidiopathic MCDs occur in the background of human immunodeficiency virus (HIV) infection especially in association with Kaposi sarcoma herpesvirus (KSHV) or HHV8 as well as in association with secondary amyloidosis. ${ }^{12,26,8,10}$

Histomorphologically CD is distinct from malignant lymphoma; however, these two lesions can coexist or even, indeed, mimic each other like in this case report. ${ }^{1,6,11-18}$ In such scenarios, immunohistochemical (IHC) evaluation of the lymph node specimen in addition to routine H\&E histopathological evaluation becomes the gold standard in arriving at a definitive diagnosis. ${ }^{1,5,11,13-15,19}$

This case is reported because of its rarity and thus should be considered in the differential diagnosis of generalized lymphadenopathy, and also to show how important IHC is in resolving diagnostic dilemmas in lymph node pathology.

\section{Case Report}

The patient is a 68-year-old woman who presented with a 1-year history of abdominal swelling and pain. These symptoms were of gradual onset and progressive over time. There was history of constitutional symptoms including low grade fever, night sweat, and weight loss. There was also a history of easy fatigue and mild unproductive cough. Clinical examination revealed moderate to severe pallor, bilateral axillary lymphadenopathy. Abdomen was mildly distended and tender with splenomegaly of approximately $14 \mathrm{~cm}$ and liver was tipped. Other systems examined were unremarkable.

Routine laboratory investigations such as full blood count showed normocytic normochromic anemia with hemoglobin level of $7.0 \mathrm{~g} / \mathrm{dL}$; renal and liver function test and other biochemical tests were normal. HIV serology was nonreactive.

Abdominal ultrasound scan showed massive splenomegaly. Computerized tomography of the abdomen revealed minimally enhancing solid presacral soft tissue mass with solitary metastasis to the spleen and lumber vertebrae suggestive of lymphoma. Bone marrow aspiration showed feature of nutritional anemia and small lymphoid follicular aggregates. Excision biopsy of both axillary lymph nodes was performed and subjected to routine histopathological and IHC evaluations.

Routine histopathological evaluation revealed complete effacement of normal lymph node architecture by polymorphous population of cells comprising mainly lymphocytes, plasma cells, and macrophages which were intimately admixed with cells having vague "Hodgkin-Reed-Sternberg" lacuna cell appearance. The background of this lesion was largely collagenized to sclerotic as well as edematous in few areas. These morphologic features were suggestive of Hodgkin Lymphoma ( - Fig. 1A,B).

Subsequent IHC evaluation showed: negative expression of CD15, CD30, EBER, and PAX5 (effectively ruling out Hodgkin-Reed-Sternberg cells); positive expression of CD5 and CD3 (showing the T-cell rich areas in the paracorti$\mathrm{cal} /$ interfollicular zones), CD23 (showing follicular dendritic cells of the germinal centers), leucocyte common antigen (showing the polymorphous [T and B cells] nature of this lesion), CD20 (showing the B cell rich areas in the follicular zones of the cortex), CD138 (showing presence of plasma cells), Kappa+Lambda (showing polyclonal immunoglobulin expression); Ki67 expression was variable (showing the variably low mitotic index); negative expression of BCL2 (ruling out follicular lymphoma). Based on these patterns of IHC expression a diagnosis of CD, plasma cell type was made (-Fig. 2).

Based on the above diagnosis, anemia was corrected with three unit of packed red cells and CHOP-21 (cyclophosphamide, adriamycin, oncovin, and prednisolone) chemotherapy was initiated. Three cycles were given, and patient responded remarkably well.

There was complete regression of the enlarged spleen and liver including the axillary lymph nodes. Patient is currently asymptomatic and on routine clinic follow-up.

\section{Discussion}

$\mathrm{CD}$ is a rare clinicopathological disease entity with various subtypes. ${ }^{1,2,5}$ The etiology of $\mathrm{CD}$ remains largely unknown; however, it is of note that some association with
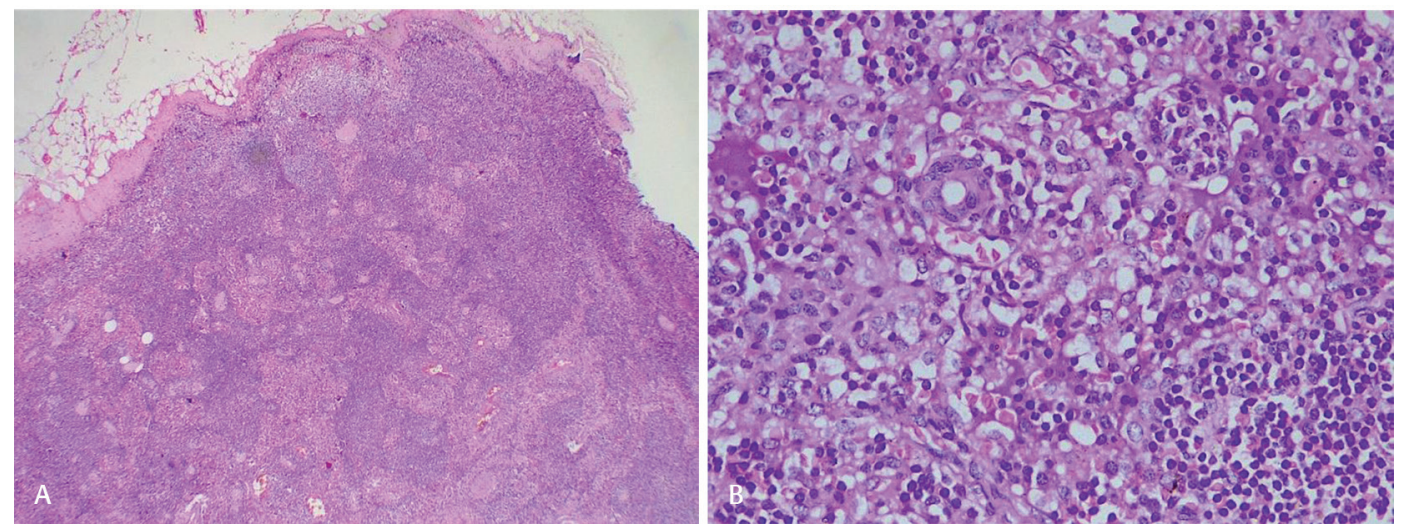

Fig. 1 (A,B) Photomicrograph of lymph node showing partial effacement of normal lymph node architecture by sheets of discohesive lymphocytes, plasma cells, histiocytes as well as thick-walled blood vessels (H\&E stain $\times 4$ and 40 , respectively). 

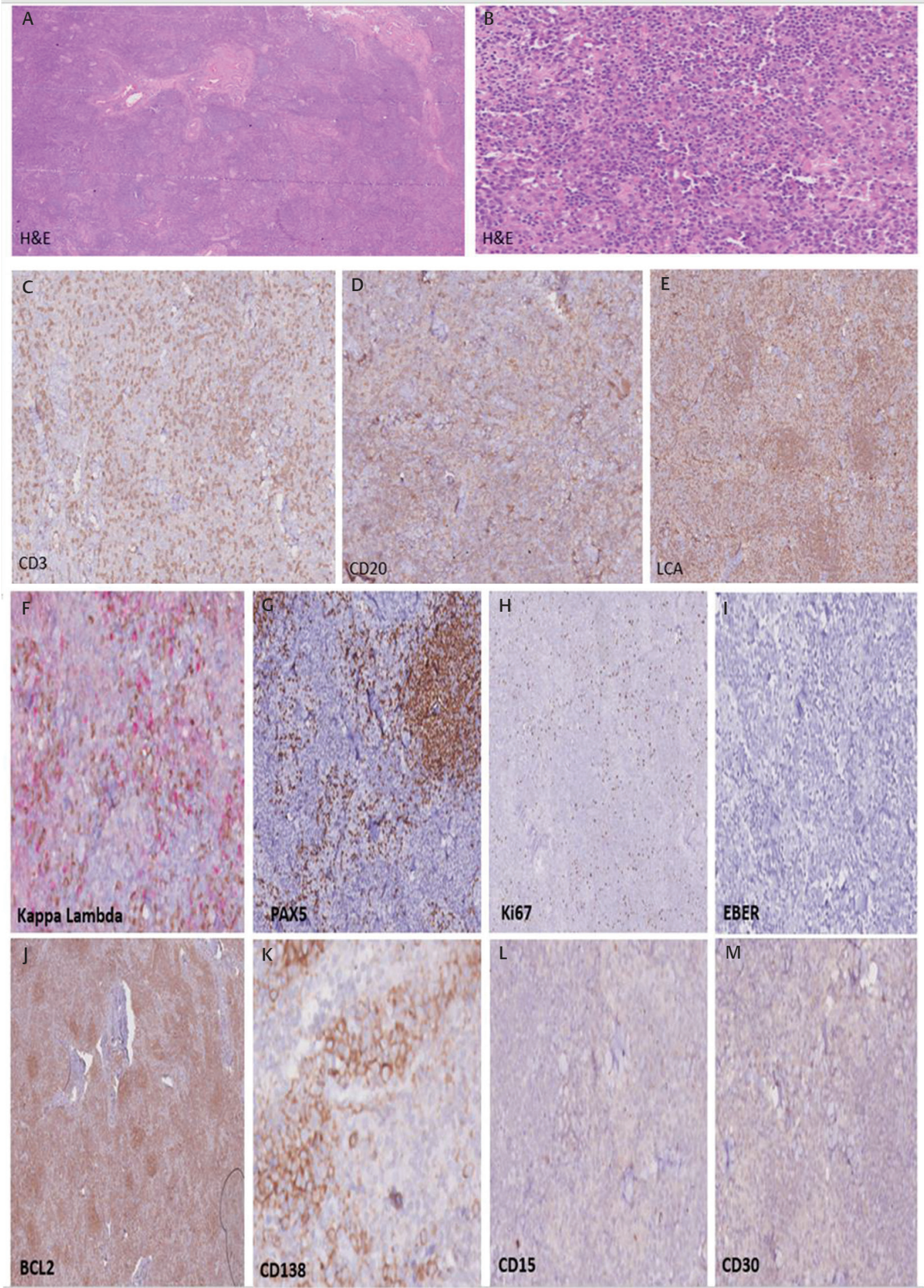

Fig. 2 (A) Histologic section, low power view, of lymph node biopsy showing complete effacement of normal lymph node by monomorphic population of lymphocytes. (B) Histologic section, intermediate power view, of lymph node biopsy showing complete effacement of normal lymph node by monomorphic population of lymphocytes. (C) Positive CD3 immunostain; this is a T-cell marker and shows expression in the paracortex; this verifies that we were dealing with a lymph node. (D) Positive CD20 immunostain; this is a B cell marker in the germinal center of the cortex, this verifies that we were dealing with a lymph node. (E) Weakly patchy positive leucocyte common antigen (LCA) or CD45 immunostain showing polymorphous nature; this rules out lymphoma (Immunohistochemical stains). (F) Kappa Lambda immunostain shows presence of polymorphous immunoglobulins. (G) PAX5 immunostain is negative for Hodgkin-Reed-Sternberg cells as well as HHV8-infected cells. (H) Ki67 immunostain shows the "Mitotic index," which is < $5 \%$ in this case, thus is not mitotically active. (I) Negative EBER immunostain, which is Epstein Barr virus (EBV) marker rules out EBV association like in Hodgkin lymphoma. (J) Negative BCL2 immunostain is a follicular lymphoma marker; thus, the negative staining rules out follicular lymphoma in this lesion. (K) Positive CD138 immunostain, which is a plasma cell marker. (L) Negative CD15 immunostain is a marker for Hodgkin-Reed-Sternberg cells (HRS), hence rules out Hodgkin lymphoma marker in this lesion. (M) Negative CD30 immunostain is a marker for Hodgkin-Reed-Sternberg (HRS) cells, hence rules out Hodgkin lymphoma marker in this lesion (immunohistochemical stains). EBV, Epstein Barr virus; EBER, EBV-encoded RNA; HHV8, human herpes virus type 8. 
immunosuppressive conditions mediated by HIV, Epstein Barr virus, KSHV (Kaposi's sarcoma-associated herpes virus) or HHV-8 (human herpes virus-8) as well as secondary amyloidosis have been found in some cases. ${ }^{2,6,8-10,20}$ The incidence of $C D$ also remains largely unknown; however, it is of note that the prevalence of $C D$ is approximately $<1$ per 100,000 worldwide. ${ }^{1,18}$ The majority of $\mathrm{CD}$ cases are adults, particularly females, with less than 100 cases reported in children (especially amongst teenage girls). ${ }^{1,18,20,21}$ These adults affected are found within the third to fourth decade for UCD and fourth to fifth decade for MCD, with UCD accounting for approximately $87 \%$ of $\mathrm{CD}$ cases. ${ }^{1,18}$

The hyaline vascular (HV) subtype of CD is characterized histomorphologically by prominent vascular proliferation and hyalinization of vessel walls admixed with variable follicular patterns such as lollipop follicles, onion skin mantle zone, and mantle zones fusion with twinning of germinal centers. $^{1,22,23}$ This subtype accounts for approximately $90 \%$ of cases and commonly seen in the localized or unicentric form (UCD) of the disease. . $^{1,4,2,23}$ It is asymptomatic in over $50 \%$ of cases, commoner among young adults and most cases are often discovered incidentally on imaging studies as a soft-tissue mass located in the neck or mediastinum and rarely in the retroperitoneum. ${ }^{4}$

The plasma cell subtype of $\mathrm{CD}$ is characterized histomorphologically by sheets of mature plasma cells within the interfollicular zones of the lymph node interspersed by surrounding larger/hyperplastic germinal center with less vascularity. ${ }^{1,11}$ The MCD form of CD is associated with this plasma cell variant. ${ }^{1}$ It usually affects older patients and clinically it is characterized by generalized lymphadenopathy, constitutional symptoms, multisystem organ involvement, and deranged laboratory findings. ${ }^{6,9,18,24}$ These constitutional symptoms are considered a consequence of elevated chemical mediators of inflammation, namely: IL6, interleukin-2 (IL2), and vascular endothelial growth factor (VEGF). ${ }^{1,6,8,9,18,20,25}$ These constitutional symptoms include: pallor, chronic diarrhea, asthenia, fever, weight loss, generalized lymphadenopathy, edema (sometimes ascites and/ or anasarca), and hepatosplenomegaly. ${ }^{1,6,8,9,18}$ The deranged laboratory findings include: anemia, thrombocytosis or thrombocytopenia, hypoalbuminemia, hypocholesterolemia, hypergammaglobulinemia, proteinuria, increased acute phase reactants (such as serum C-reactive protein), and increased chemical mediators of inflammation levels (such as IL6, IL2, and VEGF). ${ }^{6-9}$ It is of note that unlike UCD, MCD is strongly associated with secondary amyloidosis as well as immunosuppression and HHV-8 infection usually in the background of HIV infection. ${ }^{1,6,10}$ The clinical progression is often fatal due to high risk of opportunistic infections and likelihood of malignant neoplastic transformation often to Kaposi sarcoma and lymphoma., 2,618

Interestingly, our patient in this case report was diagnosed with this less common multicentric plasma cell subtype of $\mathrm{CD}$. She also presented with anemia, fever, and generalized lymphadenopathy consistent with MCD in addition to other symptoms associated with this subtype of $C D$ in agreement with $C D$ literature reviewed above. Interestingly, it is of note that though MCD is associated with HIV and HHV-8 infection, with some studies even demonstrating the presence of the HHV-8 sequence in approximately 60 to $100 \%$ of patients infected with HIV and 20 to $41 \%$ in those who were not, our index patient was HIV negative and her IHC evaluation was also negative for HHV-8 expression antibodies through the PAX-5 surrogate marker (even though direct serum HHV-8 antibodies could not be done for her).,14,15,25 The gold standard for our diagnosis in this case was a combination of routine histopathological and IHC evaluations of her cervical lymph node biopsy. The application of IHC as an ancillary technique in this case was especially critical in deciphering this case given the diagnostic dilemma initially presented by her overlapping clinical features and the initial Hodgkin's lymphoma pathologic diagnosis; thus IHC evaluation in combination with routine histopathological evaluations has been recommended as a standard method for lymph node examination especially where routine histopathological evaluation is nonspecific. . $^{1,511-15,19}$

Surgical resection of the affected lymph node is the treatment of choice for UCD, usually with no risk of recurrence; however, the MCD variant requires multimodal therapies with cytotoxic chemotherapy or CVAD (cyclophosphamide, vincristine, doxorubicin, dexamethasone, or prednisolone)/CHOP (cyclophosphamide, vincristine, doxorubicin, dexamethasone, or prednisolone), corticosteroids (dexamethasone/prednisolone), immunomodulators (lenalidomide or thalidomide, bortezomib, interferon $\alpha$ ), intravenous immunoglobulins, plasmapheresis, radiotherapy, monoclonal antibodies (tocilizumab, siltuximab, and rituximab) and autologous hematopoietic stem cell transplantation. ${ }^{2,6,9,18,20}$ In accordance with these literature our patient was successfully treated with CHOP. It is of note that a combinations of these treatment options give a better prognosis in MCD. ${ }^{9,18,20}$ The IL-6 monoclonal antibodies (tocilizumab and siltuximab) are particularly useful in the alleviation of systemic manifestation. ${ }^{6,18,20}$ Fortunately, a 5 -year survival rate of $82 \%$ have been reported in MCD, a much better prognosis when compared with lymphoma. ${ }^{26}$

\section{Conclusion}

Multicentric plasma cell subtype of $C D$ is a relatively uncommon disease entity that can present as a diagnostic dilemma because of its overlapping clinicopathological presenting features similar to malignant (Hodgkin's) lymphoma. Hence, this case report brings to the fore the importance of carrying out IHC evaluation of lymph node biopsy specimens with lymphoproliferative picture in addition to its routine histopathological examination for definitive diagnosis in view of expert management.

Conflict of Interest

None declared. 


\section{References}

1 Balakrishna J. Castleman disease pathology outlines. 2019 p. 1. Available at: http://www.pathologyoutlines.com/ topic/lymphnodescastleman.html. Accessed March 11, 2020

2 Dispenzieri A, Fajgenbaum DC. Overview of Castleman disease. Blood 2020;135(16):1353-1364

3 Castleman B, Towne V. CASE records of the Massachusetts General Hospital weekly clinicopathological exercises: case 40011 Vol. 250. N Engl J Med 1954;250(1):26-30

4 García KM, Rodriguez AM. Unicentric Castleman's disease: case report and literature review. Canc Ther Oncol Int J. 2018;9(5):1-5

5 Sevilla-Lizcano DB, Frias-Soria CL, Ortiz-Hidalgo C. Castleman disease. Histopathological and immunohistochemical analysis of 39 cases. Gac Med Mex 2017;153(5):550-558

6 Murakami M, Johkoh T, Hayashi S, et al. Clinicopathologic characteristics of 342 patients with multicentric Castleman disease in Japan. Mod Rheumatol 2020;30(5):843-851

7 Kojima M, Nakamura N, Tsukamoto N, et al. Clinical implications of idiopathic multicentric Castleman disease among Japanese: a report of 28 cases. Int J Surg Pathol 2008;16(4):391-398

8 Fujimoto S, Sakai T, Kawabata H, et al. Is TAFRO syndrome a subtype of idiopathic multicentric Castleman disease? Am J Hematol 2019;94(9):975-983

9 Liu AY, Nabel CS, Finkelman BS, et al. Idiopathic multicentric Castleman's disease: a systematic literature review. Lancet Haematol 2016;3(4):e163-e175

10 Stebbing J, Pantanowitz L, Dayyani F, Sullivan RJ, Bower M, Dezube BJ. HIV-associated multicentric Castleman's disease. Am J Hematol 2008;83(6):498-503

11 Fetica B, Pop B, Lisencu C, et al. Castleman disease. A report of six cases. Clujul Med 2014;87(3):192-197

12 Filliatre-Clement L, Busby-Venner H, Moulin C, Roth-Guepin G, Perrot A. Hodgkin lymphoma and Castleman disease: when one blood disease can hide another. Case Rep Hematol 2017; 2017(9423205):9423205

13 Maheswaran PR, Ramsay AD, Norton AJ, Roche WR. Hodgkin's disease presenting with the histological features of Castleman's disease. Histopathology 1991;18(3):249-253
14 Mohtaram A, Afif M, Sghiri T, et al. Coexistence of Hodgkin's lymphoma and Castleman's disease: a case report with successful response to chemotherapy and radiotherapy. Case Rep Oncol Med 2013;2013:487205

15 Gong S, Hijiya N. Classical Hodgkin lymphoma and Castleman disease: a rare morphologic combination. Blood 2017; 130(3):381

16 Reddy SK, Rekha JS, Jacob SE, Basu D. Diagnostic issues in a case of Hodgkin lymphoma with Castleman like features. Indian J Hematol Blood Transfus 2014;30(suppl 1) :386-389

17 Brown JR, Skarin AT. Clinical mimics of lymphoma. Oncologist 2004;9(4):406-416

18 Benmiloud S, Chaouki S, Atmani S, Hida M. Multicentric Castleman's disease in a child revealed by chronic diarrhea. Case Rep Pediatr 2015;2015:1-4

19 Rao IS. Role of immunohistochemistry in lymphoma. Indian J Med Paediatr Oncol 2010;31(4):145-147

20 Cai S, Zhong Z, Li X, Wang HX, Wang L, Zhang M. Treatment of multicentric Castleman disease through combination of tocilizumab, lenalidomide and glucocorticoids: case report. Medicine (Baltimore) 2019;98(46):e17681

21 Smith C, Lee-Miller C, Dishop MK, Cost C, Wang M, Asturias EJ. Multicentric Castleman disease presenting with fever. J Pediatr 2014;165(6):1261-1265

22 Venkataramana CG, Kini H, Saha D, et al. Histomorphologic spectrum in hyaline vascular variant of Castleman disease. J Clin Diagn Res 2017;11(8):EC01-EC04

23 Li P, Liu H, Li H, Li A, Yu G, Yin W. Hyaline vascular variant of unicentric Castleman disease of the tonsil: a case report. Diagn Pathol 2019;14(1):70

24 Waterston A, Bower M. Fifty years of multicentric Castleman's disease Vol. 43. Acta Oncol (Madr) 2004;43(8):698-704

25 Kettani M, Touiheme N, Attifi H, et al. Castle man disease: a case report and review of the literature. Pan Afr Med J 2014;19:130

26 Zhang X, Rao H, Xu X, et al. Clinical characteristics and outcomes of Castleman disease: a multicenter study of 185 Chinese patients. Cancer Sci 2018;109(1):199-206 